Article

\title{
On Periodic Solutions of Delay Differential Equations with Impulses
}

\section{Mostafa Bachar}

Department of Mathematics, College of Sciences, King Saud University, Riyadh 11451, Saudi Arabia; mbachar@ksu.edu.sa

Received: 18 February 2019; Accepted: 8 April 2019; Published: 11 April 2019

Abstract: The purpose of this paper is to study the nonlinear distributed delay differential equations with impulses effects in the vectorial regulated Banach spaces $\mathcal{R}\left([-r, 0], \mathbb{R}^{n}\right)$. The existence of the periodic solution of impulsive delay differential equations is obtained by using the Schäffer fixed point theorem in regulated space $\mathcal{R}\left([-r, 0], \mathbb{R}^{n}\right)$.

Keywords: delay differential equations; integral operator; periodic solutions

MSC: primary 06F30; 46B20; 47E10; 34K13; 34K05

\section{Introduction}

In this paper, we will investigate the existence of periodic solutions for vectorial distributed delay differential equations with impulses in regulated Banach spaces. More precisely, the prototype of this delay differential equations with impulses, is of the form

$$
\begin{aligned}
\frac{d x(t)}{d t} & =-\lambda x(t)+f\left(t, x_{t}\right), \text { a.e. } t \in[0, \omega+\tau], \lambda>0, \omega>0, \\
x\left(t_{j}\right) & =x\left(t_{j}^{-}\right), \text {and } x\left(t_{j}^{+}\right)-x\left(t_{j}\right)=h_{j}\left(x\left(t_{j}\right)\right), \forall j=1, \ldots, l, \\
x_{0}(\theta) & =\varphi(\theta), \theta \in[-\tau, 0],
\end{aligned}
$$

with $x_{t}(\theta)=x(t+\theta), \theta \in[-\tau, 0], \tau>0$ and where $x$ and $\varphi$ are $\mathbb{R}^{n}$-valued functions on $[-\tau, \omega]$, and $[-\tau, 0]$, respectively. The Equation (1) is a nonlinear delay differential equation. More details about this type of equations can be found in [1]. Moreover, we assume that

(i) $h_{j} \in C\left(\mathbb{R}^{n}, \mathbb{R}^{n}\right), j=1, \ldots, l$,

(ii) $\left\{t_{1}, t_{2}, \cdots, t_{l}\right\}$ is an increasing family of strictly positive real numbers,

(iii) there exist $\delta>0$ and $T<\infty$, such that for any $j=1, \ldots, l-1$, we have

$$
0<\delta \leq t_{j+1}-t_{j} \leq T<\infty
$$

We call (2) the impulses equation where, $x\left(t_{j}^{-}\right)$(resp. $x\left(t_{j}^{+}\right)$) denotes the limit from the left (resp. from the right) of $x(t)$, as $t$ tends to $t_{j}$. This type of differential equations without delay was initiated in 1960's by Milman and Myshkis [2,3]. This problem started to be popular mostly in Eastern Europe in the years 1960-1970, with special attention during the seventies of the last century. Later on, several investigations and important monographs appeared with more details, which show the importance of studying such systems, see for example [4-11]. In recent years, many investigations have arisen with applications to life sciences, such that the periodic treatment of some biomedical applications, where the impulses correspond to administration of a drug treatment at certain given times [12-15]. However, comparatively speaking, not much has been done in the study of impulsive functional 
differential equations in regulated vectorial space, taking into account the general theory of functional analysis and having an acceptable hypothesis that can be used in real life applications, see [12] for more details.

Let us first introduce for each $\tau>0$, the regulated Banach space $\mathcal{R}=\mathcal{R}\left([-\tau, 0], \mathbb{R}^{n}\right)$, given by:

$$
\mathcal{R}=\left\{\varphi:[-\tau, 0] \rightarrow \mathbb{R}^{n}: \varphi \text { has left and right limits at every points of }[-\tau, 0]\right\},
$$

endowed with the following norm

$$
\|\varphi\|_{\mathcal{R}}=\sup _{\theta \in[-\tau, 0]}\|\varphi(\theta)\| .
$$

We will make the following assumptions

(I) The map $f:[0, \omega+\tau] \times \mathbb{R}^{n} \rightarrow \mathbb{R}^{n}, \omega>0$, satisfies

- $\|f(t, \varphi)-f(t, \psi)\|_{\mathcal{R}} \leq K\|\varphi-\psi\|_{\mathcal{R}}, \forall t \in[0, \omega+\tau], \varphi, \psi \in \mathcal{R}$,

- $\quad \exists M>0,\|f(t, 0)\|_{\mathcal{R}} \leq M, \forall t \in[0, \omega+\tau]$.

(II) For each regulated map $x:[a, b] \rightarrow \mathbb{R}^{n}$, with $b-a>\tau$, we assume that the map $t \rightarrow f\left(t, x_{t}\right)$ is measurable over $[a+\tau, b]$.

(III) For each $j=1, \ldots, l, h_{j}: \mathbb{R}^{n} \rightarrow \mathbb{R}^{n}$ is a continuous map.

We set the initial value problem as follows

Problem 1. Let $\varphi$ be an element of $\mathcal{R}$. We want to find a function $x$ defined on $[-\tau, \omega+\tau]$ such that $x$ satisfies (1)-(3).

We consider the nonlinear impulsive delay differential equation in $\mathcal{R}$ as

$$
\left\{\begin{aligned}
\frac{d x(t)}{d t} & =-\lambda x(t)+f\left(t, x_{t}\right), \text { a.e. } t \in[0, \omega+\tau], \lambda>0, \omega>0, \\
x\left(t_{j}\right) & =x\left(t_{j}^{-}\right), \text {and } x\left(t_{j}^{+}\right)-x\left(t_{j}\right)=h_{j}\left(x\left(t_{j}\right)\right), \forall j=1, \ldots, l, \\
x_{0}(\theta) & =\varphi(\theta), \theta \in[-\tau, 0] \text { and } x\left(0^{+}\right)=\xi \in \mathbb{R}^{n} .
\end{aligned}\right.
$$

The aim of this paper is to extend the main results related to the existence of the $\omega$-periodic solutions for ordinary differential equations with impulses presented by Li et al. [16] and Nieto [17]. These papers contain references which provide additional reading on this topic, i.e., differential equations with impulses by using the fixed point theory.

\section{Existence and Uniqueness of Solution}

Let us start first by introducing some related definitions and lemmas.

Definition 1. A function $x:[-\tau, \omega+\tau] \rightarrow \mathbb{R}^{n}$ is called a solution of (1)-(3) if:

1. $x$ is absolutely continuous with respect to the Lebesgue measure;

2. $x$ is differentiable on the complement of a countable subset of $[0, \omega+\tau]$, and satisfies Equation (1) whenever $\frac{d x(t)}{d t}$ and the right hand side of (1) are defined on $[0, \omega+\tau]$;

3. $x$ satisfies (2) at each point $t_{j}, t_{j} \geq 0, \forall j=1, \ldots, l$, and the initial value function satisfies (3).

Lemma 1. Let $f:[0, \omega+\tau] \times \mathcal{R} \rightarrow \mathbb{R}^{n}$ be a map satisfying (I) and (II) and $t_{1} \in[0, \omega+\tau]$. Then, for each $(\varphi, \xi) \in \mathcal{R} \times \mathbb{R}^{n}$, the problem

$$
\begin{aligned}
\frac{d x(t)}{d t} & =-\lambda x(t)+f\left(t, x_{t}\right), \text { a.e. } t \in\left[0, t_{1}\right] \\
\left(x_{0}, x\left(0^{+}\right)\right) & =(\varphi, \xi) \in \mathcal{R} \times \mathbb{R}^{n},
\end{aligned}
$$


has a unique solution.

Proof. We set $S=\left\{y \in C\left(\left[0, t_{1}\right], \mathbb{R}^{n}\right), y(0)=x\left(0^{+}\right)=\xi\right\}$. Let us define the operator $T$ by

$$
T(x)(t)=\xi+\int_{0}^{t}\left(f\left(s, x_{s}\right)-\lambda x(s)\right) d s, 0 \leq t \leq t_{1} .
$$

For each $y \in S$, we consider the Nemytski operator $F$, defined by

$$
F(y)(t)=f\left(t, z_{t}\right)
$$

where

$$
z_{t}(\theta)= \begin{cases}y(t+\theta), & \text { if } t+\theta \geq 0 \\ \varphi(t+\theta), & \text { if } t+\theta \leq 0\end{cases}
$$

Then, we get

$$
T(y)(t)=\xi+\int_{0}^{t}(F(y)(s)-\lambda y(s)) d s .
$$

Define, the norm of any function $y$ in $S$ by

$$
\|y\|_{S}=\sup _{0 \leq t \leq t_{1}}\left\{\|y(t)\| e^{-\rho t}\right\}
$$

where $\rho$ is a fixed positive constant greater than $K+\lambda$. We have for each $y_{1}(t)$ and $y_{2}(t)$ in $S$,

$$
\begin{aligned}
\left\|T\left(y_{1}\right)(t)-T\left(y_{2}\right)(t)\right\| & \leq(K+\lambda) \int_{0}^{t}\left\|y_{1}(s)-y_{2}(s)\right\| d s \\
& \leq(K+\lambda) \int_{0}^{t} e^{\rho s-\rho s}\left\|y_{1}(s)-y_{2}(s)\right\| d s, \\
& \leq(K+\lambda)\left\|y_{1}-y_{2}\right\|_{s} \int_{0}^{t} e^{\rho s} d s \\
& \leq \frac{(K+\lambda)}{\rho}\left\|y_{1}-y_{2}\right\|_{s} e^{\rho t}
\end{aligned}
$$

and hence

$$
\left\|T\left(y_{1}\right)-T\left(y_{2}\right)\right\|_{S} \leq \frac{(K+\lambda)}{\rho}\left\|y_{1}-y_{2}\right\|_{S} .
$$

Since $\frac{K+\lambda}{\rho}<1$, then, $T$ is a contraction on $S$, and the result follows immediately.

Lemma 2. [18] Let $f:[0, \omega+\tau] \times \mathcal{R} \rightarrow \mathbb{R}^{n}$ be a map satisfying (I) and (II) and $h_{j}$, for $j=1, \cdots, l$, satisfy the condition (III). Then the problem (1)-(3) has a unique solution.

Proof. The proof follows by using the last lemma.

Lemma 3. [18] Under the assumptions (I) and (II), if $x(\varphi)(t)$ is the unique solution of (4) and (5), then one has:

$$
\|x(\varphi)(t)\| \leq e^{K t}\left(\|\varphi\|+\int_{0}^{\omega}\|f(s, 0)\| d s\right) .
$$

The next Lemma, gives a similar, key representation formula for the solutions of the delay differential equations with impulses (1)-(3) in regulated Banach space $\mathcal{R}$, see [4] for more details. 
Lemma 4. The problem (1)-(3) can be written as

$$
\begin{aligned}
x_{t}= & \varphi_{t}^{0}+H_{t}^{0} \otimes\left(\left(\xi e^{-\lambda \max (0, \bullet)}\right)_{t}-\varphi(0)\right) \\
& +\left(\int_{0}^{\max (0, \bullet)} f\left(s, x_{s}\right) e^{-\lambda(\bullet-s)} d s+\sum_{0 \leq t_{j}<\bullet} e^{-\lambda\left(\bullet-t_{j}\right)} u_{j}\right)_{t^{\prime}}
\end{aligned}
$$

where

$$
\varphi^{0}(\theta)= \begin{cases}\varphi(\theta), & \text { if } \theta \leq 0 \\ \varphi(0), & \text { if } \theta>0\end{cases}
$$

$H^{0}$ is the Heaviside function

$$
H^{0}(\theta)= \begin{cases}0, & \text { if } \theta \leq 0 \\ 1, & \text { if } \theta>0\end{cases}
$$

and the sequence

$$
u_{k}=x\left(t_{k}^{+}\right)-x\left(t_{k}\right), k \geq 1
$$

is determined by the following non-autonomous recurrence equation

$$
u_{k}=h_{k}\left(\xi e^{-\lambda t_{k}}+\int_{0}^{t_{k}} f\left(s, x_{s}\right) e^{-\lambda\left(t_{k}-s\right)} d s+\sum_{0 \leq t_{j}<t_{k}} e^{-\lambda\left(t_{k}-t_{j}\right)} u_{j}\right), k \geq 1 \text {, }
$$

starting from

$$
u_{1}=h_{1}\left(\xi e^{-\lambda t_{1}}+\int_{0}^{t_{1}} f\left(s, x_{s}\right) e^{-\lambda\left(t_{1}-s\right)} d s\right) .
$$

Proof. Let us consider $z(t)=e^{\lambda t} x(t), \forall t \in[0, \omega+\tau]$, then the problem (1)-(3) becomes

$$
\begin{aligned}
\frac{d z(t)}{d t} & =f\left(t, e^{-\lambda(t+\theta)} z_{t}\right) e^{\lambda t}, \text { a.e. } t \in[0, \omega+\tau], \lambda>0, \omega>0, \\
z\left(t_{j}\right) & =z\left(t_{j}^{-}\right), \text {and } z\left(t_{j}^{+}\right)-z\left(t_{j}\right)=e^{\lambda t_{j}} h_{j}\left(e^{-\lambda t_{j}} z\left(t_{j}\right)\right), \forall j=1, \ldots, l, \\
z_{0}(\theta) & =e^{\lambda \theta} \varphi(\theta)=\widetilde{\varphi}(\theta), \theta \in[-\tau, 0], \text { and } z\left(0^{+}\right)=\xi \in \mathbb{R}^{n} .
\end{aligned}
$$

Let us consider $t \in\left[t_{j}, t_{j+1}\right), j=1, \ldots, l-1$, with $t_{0}=0$, then we get

$$
z(t)=z\left(t_{j}^{+}\right)+\int_{t_{j}}^{t} f\left(s, e^{-\lambda(s+\theta)} z_{s}\right) e^{\lambda s} d s .
$$

By passing to the limit as $t$ goes to $t_{j}^{-}$, and by solving the delay differential Equation (14) on the interval $\left[t_{j-1}, t_{j}\right)$, we have

$$
z\left(t_{j}\right)=z\left(t_{j-1}^{+}\right)+\int_{t_{j-1}}^{t_{j}} f\left(s, e^{-\lambda(s+\theta)} z_{s}\right) e^{\lambda s} d s
$$

Then, by taking into account the impulses condition (15), we have

$$
z(t)=z\left(t_{j-1}^{+}\right)+\int_{t_{j-1}}^{t} f\left(s, e^{-\lambda(s+\theta)} z_{s}\right) e^{\lambda s} d s+e^{\lambda t_{j}} h_{j}\left(e^{-\lambda t_{j}} z\left(t_{j}\right)\right),
$$


for all $t \in\left[t_{j}, t_{j+1}\right)$, for $j=1, \cdots, l-1$. Consequently, we can rewrite the last equations in more general form for all $t>0$

$$
z(t)=\xi+\int_{0}^{t} f\left(s, e^{-\lambda(s+\theta)} z_{s}\right) e^{\lambda s} d s+\sum_{0 \leq t_{j}<t} e^{\lambda t_{j}} u_{j}, t \notin\left\{t_{k}\right\}_{k \geq 1}
$$

where $z\left(0^{+}\right)=x\left(0^{+}\right)=\xi$, and

$$
u_{k}=z\left(t_{k}^{+}\right)-z\left(t_{k}\right)=h_{k}\left(e^{-\lambda t_{k}} z\left(t_{k}\right)\right), k \geq 1
$$

Now, we will try to involve the $u_{k}^{\prime} s$. To this end, we will take the limit from the left of the Formula (17) as $t$ tends to $t_{k}>0$, we obtain

$$
z\left(t_{k}\right)=\xi+\int_{0}^{t_{k}} f\left(s, e^{-\lambda(s+\theta)} z_{s}\right) e^{\lambda s} d s+\sum_{0 \leq t_{j}<t_{k}} e^{\lambda t_{j}} u_{j}
$$

Substituting the last expression into (18), we have

$$
u_{k}=h_{k}\left(e^{-\lambda t_{k}} \xi+\int_{0}^{t_{k}} f\left(s, e^{-\lambda(s+\theta)} z_{s}\right) e^{\lambda\left(s-t_{k}\right)} d s+\sum_{0 \leq t_{j}<t_{k}} e^{\lambda\left(t_{j}-t_{k}\right)} u_{j}\right) .
$$

In particular, we have $\left\{j: 0 \leq t_{j}<t_{1}\right\}=\varnothing$, and therefore

$$
u_{1}=h_{1}\left(e^{-\lambda t_{1}} \xi+\int_{0}^{t_{1}} f\left(s, e^{-\lambda(s+\theta)} z_{s}\right) e^{\lambda s} d s\right) .
$$

By using, the Equation (16), we can rewrite the Equation (17) as

$$
\begin{aligned}
z_{t}(\theta)= & \xi+\int_{0}^{t+\theta} f\left(s, e^{-\lambda(s+\theta)} z_{s}\right) e^{\lambda s} d s \\
& +\sum_{0 \leq t_{j}<t+\theta} e^{\lambda t_{j}} u_{j}, t+\theta \notin\left\{t_{k}\right\}_{k \geq 0}, \text { and } t+\theta \geq 0,
\end{aligned}
$$

and by using $x(t)=e^{-\lambda t} z(t)$, we have for $t+\theta \notin\left\{t_{k}\right\}_{k \geq 1}$, and $t+\theta \geq 0$

$$
x_{t}(\varphi(\theta))=\xi e^{-\lambda(t+\theta)}+\int_{0}^{t+\theta} f\left(s, x_{s}\right) e^{-\lambda(t+\theta-s)} d s+\sum_{0 \leq t_{j}<t+\theta} e^{-\lambda\left(t+\theta-t_{j}\right)} u_{j} .
$$

Using (12) and (13), we get

$$
\begin{aligned}
x_{t}(\varphi)= & \varphi_{t}^{0}+H_{t}^{0} \otimes\left(\left(\xi e^{-\lambda \max (0, \bullet)}\right)_{t}-\varphi(0)\right) \\
& +\left(\int_{0}^{\max (0, \bullet)} f\left(s, x_{s}\right) e^{-\lambda(\bullet-s)} d s+\sum_{0 \leq t_{j}<\bullet} e^{-\lambda\left(\bullet-t_{j}\right)} u_{j}\right)_{t^{\prime}}
\end{aligned}
$$

where

$$
u_{k}=h_{k}\left(\xi e^{-\lambda t_{k}}+\int_{0}^{t_{k}} f\left(s, x_{s}\right) e^{-\lambda\left(t_{k}-s\right)} d s+\sum_{0 \leq t_{j}<t_{k}} e^{-\lambda\left(t_{k}-t_{j}\right)} u_{j}\right), k \geq 1 .
$$

starting from

$$
u_{1}=h_{1}\left(\xi e^{-\lambda t_{1}}+\int_{0}^{t_{1}} f\left(s, x_{s}\right) e^{-\lambda\left(t_{1}-s\right)} d s\right) .
$$


Remark 1. Taking into account the conditions (II)-(III), we have $u_{t} \in \mathcal{R}, \forall t \in[0, \omega+\tau]$, and $t \rightarrow x_{t}$ is a regulated function, because the functions $t \rightarrow \varphi_{t}^{0}$, and $t \rightarrow H_{t}^{0}$ are regulated.

In the next section, we will investigate the existence of the periodic solution(s) for the delay differential equation with impulses (1)-(3) using Schäffer's fixed point theorem [19].

\section{Existence of Periodic Solutions}

Let us consider the Poincaré operator, given by:

$$
\begin{aligned}
J: \mathcal{R} & \rightarrow \mathcal{R} \\
\varphi & \rightarrow x_{\omega}(\varphi),
\end{aligned}
$$

where $x_{\omega}(\varphi)$ is the solution of the delay differential equation with impulses (1)-(3). It is clear that if the Poincaré operator $J$ admit a fixed point, then (1)-(3) has a $\omega$-periodic solution. The following lemma is useful to prove the main theorem.

Lemma 5. The problem (1)-(3) has a $\omega$-periodic solution in $\mathcal{R}$ if and only if the integral equation

$$
x_{t}(\varphi)(\theta)= \begin{cases}e^{-\lambda \theta} \int_{t+\theta}^{t+\omega+\theta} G(t, s) f\left(s, x_{s}\right) d s+e^{-\lambda \theta} \sum_{t+\theta \leq t_{j}<t+\omega+\theta} G\left(t, t_{j}\right) u_{j}, & \text { if } 0 \leq t+\theta \leq \omega, \\ \varphi(t+\theta), & \text { if }-\tau \leq t+\theta \leq 0,\end{cases}
$$

has a solution $\forall t \in[0, \omega+\tau]$ and $\omega \geq \tau$, where

$$
G(t, s)=\frac{e^{-\lambda(t-s)}}{e^{\lambda \omega}-1}
$$

and the sequence

$$
u_{k}=x\left(t_{k}^{+}\right)-x\left(t_{k}\right), k \geq 1
$$

is determined by the following non-autonomous recurrence equation

$$
u_{k}=h_{k}\left(\int_{t_{k}}^{t_{k}+\omega} G(t, s) f\left(s, x_{s}\right) d s+\sum_{t_{k} \leq t_{j}<t_{k}+\omega} G\left(t, t_{j}\right) u_{j}\right), k \geq 1,
$$

starting from

$$
u_{1}=h_{1}\left(\xi e^{-\lambda t_{1}}+\int_{0}^{t_{1}} f\left(s, x_{s}\right) e^{-\lambda\left(t_{1}-s\right)} d s\right)
$$

Proof. Using the expression (19) for $t+\omega+\theta$, where $t \geq 0$, and $\omega \geq \tau$, we have for all $t+\theta \geq 0$

$$
\begin{aligned}
z_{t+\omega}(\theta)= & \xi+\int_{0}^{t+\omega+\theta} f\left(s, e^{-\lambda(s+\theta)} z_{s}\right) e^{\lambda s} d s+\sum_{0 \leq t_{j}<t+\omega+\theta} e^{\lambda t_{j}} u_{j}, \\
= & \xi+\int_{0}^{t+\theta} f\left(s, e^{-\lambda(s+\theta)} z_{s}\right) e^{\lambda s} d s+\sum_{0 \leq t_{j}<t+\theta} e^{\lambda t_{j}} u_{j} \\
& +\int_{t+\theta}^{t+\omega+\theta} f\left(s, e^{-\lambda(s+\theta)} z_{s}\right) e^{\lambda s} d s+\sum_{t+\theta \leq t_{j}<t+\omega+\theta} e^{\lambda t_{j}} u_{j}, \\
= & z_{t}(\theta)+\int_{t+\theta}^{t+\omega+\theta} f\left(s, e^{-\lambda(s+\theta)} z_{s}\right) e^{\lambda s} d s+\sum_{t+\theta \leq t_{j}<t+\omega+\theta} e^{\lambda t_{j}} u_{j},
\end{aligned}
$$


and, by using the $\omega$-periodic condition $z_{t+\omega}(\theta)=e^{\lambda \omega} z_{t}(\theta)$, we get

$$
z_{t}(\theta)=\frac{1}{e^{\lambda \omega}-1} \int_{t+\theta}^{t+\omega+\theta} f\left(s, e^{-\lambda(s+\theta)} z_{s}\right) e^{\lambda s} d s+\frac{1}{e^{\lambda \omega}-1} \sum_{t+\theta \leq t_{j}<t+\omega+\theta} e^{\lambda t_{j}} u_{j} .
$$

Therefore, using $z_{t}(\theta)=e^{\lambda(t+\theta)} x_{t}(\theta)$, we have

$$
x_{t}(\theta)=e^{-\lambda \theta} \int_{t+\theta}^{t+\omega+\theta} G(t, s) f\left(s, x_{s}\right) d s+e^{-\lambda \theta} \sum_{t+\theta \leq t_{j}<t+\omega+\theta} G\left(t, t_{j}\right) u_{j},
$$

where

$$
G(t, s)=\frac{e^{-\lambda(t-s)}}{e^{\lambda \omega}-1}
$$

Then

$$
u_{k}=h_{k}\left(\int_{t_{k}}^{t_{k}+\omega} G(t, s) f\left(s, x_{s}\right) d s+\sum_{t_{k} \leq t_{j}<t_{k}+\omega} G\left(t, t_{j}\right) u_{j}\right), k \geq 1,
$$

starting from

$$
u_{1}=h_{1}\left(\xi e^{-\lambda t_{1}}+\int_{0}^{t_{1}} f\left(s, x_{s}\right) e^{-\lambda\left(t_{1}-s\right)} d s\right) .
$$

Example 1. Let us consider the scalar delay differential equation with impulses:

$$
\begin{aligned}
\frac{d x(t)}{d t} & =-\lambda x(t)+f(t, x(t-\tau)), \text { a.e. } t \in[0,2 \tau], \\
x(\tau) & =x\left(\tau^{-}\right), \text {and } x\left(\tau^{+}\right)-x(\tau)=c x(\tau), \\
x(\theta) & =\varphi(\theta), \theta \in[-\tau, 0],
\end{aligned}
$$

where $f:[0,2 \tau] \times \mathcal{R} \rightarrow \mathbb{R}^{n}$ is a map satisfying (II). Let us investigate the existence of the $\tau$-periodic solution of (22)-(24) such that $x_{t+\tau}(-\tau)=x_{t}(-\tau), \tau \leq t \leq 2 \tau$. The solution of the delay differential Equations (22)-(24), can be written as

$$
x(t)= \begin{cases}\varphi(\theta), & \text { if }-\tau \leq t \leq 0, \\ \varphi(0) e^{-\lambda t}+\int_{0}^{t} e^{-\lambda(t-s)} f(s, x(s-\tau)) d s, & \text { if } 0 \leq t \leq \tau, \\ x\left(\tau^{+}\right) e^{-\lambda(t-\tau)}+\int_{\tau}^{t} e^{-\lambda(t-s)} f(s, x(s-\tau)) d s, & \text { if } \tau<t \leq 2 \tau .\end{cases}
$$

Using (23), we get

$$
\begin{aligned}
x\left(\tau^{+}\right) & =x(\tau)+c x(\tau), \\
& =(c+1) x(0) e^{-\lambda \tau}+(c+1) \int_{0}^{\tau} e^{-\lambda(\tau-s)} f(s, x(s-\tau)) d s .
\end{aligned}
$$

Therefore, if $\tau \leq t<2 \tau$, we have 


$$
\begin{aligned}
x(t)= & \left((c+1) \varphi(0) e^{-\lambda \tau}+(c+1) \int_{0}^{\tau} e^{-\lambda(\tau-s)} f(s, x(s-\tau)) d s\right) e^{-\lambda(t-\tau)} \\
& +\int_{\tau}^{t} e^{-\lambda(t-s)} f(s, x(s-\tau)) d s, \\
= & (c+1) \varphi(0) e^{-\lambda \tau} e^{-\lambda(t-\tau)}+(c+1) e^{-\lambda \tau} \int_{0}^{t-\tau} e^{-\lambda(t-\tau-s)} f(s, x(s-\tau)) d s \\
& +(c+1) e^{-\lambda \tau} \int_{t-\tau}^{\tau} e^{-\lambda(t-\tau-s)} f(s, x(s-\tau)) d s+\int_{\tau}^{t} e^{-\lambda(t-s)} f(s, x(s-\tau)) d s, \\
= & (c+1) e^{-\lambda \tau}\left(x(t-\tau)+\int_{t-\tau}^{\tau} e^{-\lambda(t-\tau-s)} f(s, x(s-\tau)) d s\right)+\int_{\tau}^{t} e^{-\lambda(t-s)} f(s, x(s-\tau)) d s,
\end{aligned}
$$

which implies

$$
\begin{aligned}
x_{t+\tau}(-\tau)= & (c+1) e^{-\lambda \tau}\left(x_{t}(-\tau)+\int_{t-\tau}^{\tau} e^{-\lambda(t-\tau-s)} f(s, x(s-\tau)) d s\right) \\
& +\int_{\tau}^{t} e^{-\lambda(t-s)} f(s, x(s-\tau)) d s .
\end{aligned}
$$

Then, we have three cases.

(1) If $1-(c+1) e^{-\lambda \tau} \neq 0$, then, we have the existence and uniqueness of a $\tau$-periodic solution.

(2) If $1-(c+1) e^{-\lambda \tau}=0$, and

$$
\int_{t-\tau}^{\tau} e^{-\lambda(t-\tau-s)} f(s, x(s-\tau)) d s+\int_{\tau}^{t} e^{-\lambda(t-s)} f(s, x(s-\tau)) d s=0,
$$

then, we have the existence of infinitely many $\tau$-periodic solutions.

(3) If $1-(c+1) e^{-\lambda \tau}=0$, and

$$
\int_{t-\tau}^{\tau} e^{-\lambda(t-\tau-s)} f(s, x(s-\tau)) d s+\int_{\tau}^{t} e^{-\lambda(t-s)} f(s, x(s-\tau)) d s \neq 0,
$$

then, there exists no $\tau$-periodic solution.

Now, we can consider for each $t \geq-\tau$ and $\omega \geq \tau$, the Poincaré operator $J: \mathcal{R} \rightarrow \mathcal{R}$ defined by

$$
J \varphi=\left(e^{-\lambda(\bullet-t)} \int_{\bullet}^{\bullet+\omega} G(t, s) f(s, \varphi) d s+e^{-\lambda(\bullet-t)} \sum_{\bullet \leq t_{j}<\bullet+\omega} G\left(t, t_{j}\right) u_{j}\right)_{t^{\prime}}
$$

where

$$
u_{k}=h_{k}\left(\int_{t_{k}}^{t_{k}+\omega} G(t, s) f\left(s, x_{s}\right) d s+\sum_{t_{k} \leq t_{j}<t_{k}+\omega} G\left(t, t_{j}\right) u_{j}\right), k \geq 2,
$$

and, starting from

$$
u_{1}=h_{1}\left(\xi e^{-\lambda t_{1}}+\int_{0}^{t_{1}} f\left(s, x_{s}\right) e^{-\lambda\left(t_{1}-s\right)} d s\right) .
$$

It is clear, that, the $\omega$-periodic solutions in $\mathcal{R}$ of (1)-(3) are exactly the fixed points of the Poincare operator $J$, i.e., $J \varphi=\varphi$.

The following theorem, is known as the Schäffer's fixed point theorem [19], which can be found for example in Deimling's book [20]. 
Theorem 1. [19-22] Let $X$ be a normed space, $\mathcal{F}$ a continuous mapping of $X$ into $X$, such that the closure of $\mathcal{F}(B)$ is compact for any bounded subset $B$ of $X$. Then either:

(i) the equation $x=\lambda \mathcal{F} x$ has a solution for $\lambda=1$, or

(ii) the set of all such solutions $x$, for $0<\lambda<1$, is unbounded.

Before, we state the main theorem of our work, we will need the following lemma.

Lemma 6. Let $f:[0, \omega+\tau] \times \mathcal{R} \rightarrow \mathbb{R}^{n}$ be a map satisfying (I) and (II), where $\omega \geq \tau$, and $h_{j}, j=1, \ldots, l$ are bounded and satisfy the condition (III). Then, the Poincaré operator $J: \mathcal{R} \rightarrow \mathcal{R}$ is completely continuous.

Proof. Let $B \subset \mathcal{R}$ be a bounded set and $\varphi \in B$. Then by using the condition (I), we have

$$
\|f(t, \varphi)\|_{\mathcal{R}} \leq\|f(t, 0)\|_{\mathcal{R}}+\|f(t, \varphi)-f(t, 0)\|_{\mathcal{R}} \leq M+K\|\varphi\|_{\mathcal{R}}<\infty .
$$

Therefore, there exist two constants $\widetilde{M}$ and $\bar{M}$ such that

$$
\begin{aligned}
\|J \varphi(\theta)\| & =\left\|e^{-\lambda \theta} \int_{t+\theta}^{t+\theta+\omega} f(s, \varphi) G(t, s) d s+e^{-\lambda \theta} \sum_{t+\theta \leq t_{j}<t+\theta+\omega} G\left(t, t_{j}\right) u_{j}\right\|, \\
& \leq\left\|e^{-\lambda \theta} \int_{t+\theta}^{t+\theta+\omega} f(s, \varphi) G(t, s) d s\right\|+e^{\lambda r}\left\|\sum_{t+\theta \leq t_{j}<t+\theta+\omega} G\left(t, t_{j}\right) u_{j}\right\|, \\
& \leq e^{\lambda \tau} \omega \widetilde{M}+e^{\lambda r} \sum_{t+\theta \leq t_{j}<t+\theta+\omega} \bar{M},
\end{aligned}
$$

where

$$
\left\|u_{k}\right\|=\left\|h_{k}\left(\int_{t_{k}}^{t_{k}+\omega} G(t, s) f\left(s, x_{s}\right) d s+\sum_{t_{k} \leq t_{j}<t_{k}+\omega} G\left(t, t_{j}\right) u_{j}\right)\right\|<\infty, k \geq 2
$$

and starting from

$$
\left\|u_{1}\right\|=\left\|h_{1}\left(\xi e^{-\lambda t_{1}}+\int_{0}^{t_{1}} f\left(s, x_{s}\right) e^{-\lambda\left(t_{1}-s\right)} d s\right)\right\|<\infty,
$$

and, we have

$$
\|J \varphi\|_{\mathcal{R}} \leq e^{\lambda \tau} \omega \tilde{M}+e^{\lambda r} \bar{M} \sum_{t+\theta \leq t_{j}<t+\theta+\omega} 1
$$

which imply that $J(B)$ is uniformly bounded. For each $t \geq 0$, there exists $n \in \mathbb{N}^{*}$ such that $t \in\left[t_{n}, t_{n+1}\right)$, and for any $\theta, \widetilde{\theta} \in[-r, 0]$, one can obtain for any $\varphi \in B$

$$
\begin{aligned}
\|J \varphi(\theta)-J \varphi(\widetilde{\theta})\| \leq & \left\|e^{-\lambda \theta} \int_{t+\theta}^{t+\theta+\omega} f(s, \varphi) G(t, s) d s-e^{-\lambda \theta} \int_{t+\widetilde{\theta}}^{t+\widetilde{\theta}+\omega} f(s, \varphi) G(t, s) d s\right\| \\
& +\left\|e^{-\lambda \theta} \sum_{t+\theta \leq t_{j}<t+\theta+\omega} G\left(t, t_{j}\right) u_{j}-e^{-\lambda \theta} \sum_{t+\widetilde{\theta} \leq t_{j}<t+\widetilde{\theta}+\omega} G\left(t, t_{j}\right) u_{j}\right\|, \\
\leq & \frac{e^{\lambda(r+\omega)}(M+K\|\varphi\|)}{1-e^{-\lambda \omega}}\left\|\int_{t+\theta}^{t+\theta+\omega} e^{-\lambda(t-s)} d s-\int_{t+\widetilde{\theta}}^{t+\widetilde{\theta}+\omega} e^{-\lambda(t-s)} d s\right\| \\
& +\frac{e^{\lambda(r+\omega)}}{1-e^{-\lambda \omega}}\left\|\sum_{t+\theta \leq t_{j}<t+\theta+\omega} u_{j}-\sum_{t+\tilde{\theta} \leq t_{j}<t+\widetilde{\theta}+\omega} u_{j}\right\| .
\end{aligned}
$$


Therefore, for each $t \in\left[t_{n}, t_{n+1}\right)$, we will have as $|\theta-\widetilde{\theta}|$ goes to $0,\|J \varphi(\theta)-J \varphi(\widetilde{\theta})\|$ goes to 0 , which imply that the Poincaré operator $J(B)$ is equicontinuous. Using Arzelà-Ascoli's theorem, we conclude that the Poincaré operator $J$ is completely continuous.

Now, we are ready to state the main result of our work, related to the existence of $\omega$-periodic solution(s) of (1)-(3).

Theorem 2. Let $f:[0, \omega+\tau] \times \mathcal{R} \rightarrow \mathbb{R}^{n}$ be a map satisfying (I) and (II), where $\omega \geq \tau$, and $h_{j}, j=1, \ldots, l$ are bounded and satisfy the condition (III). Then, the nonlinear impulsive problem (1)-(3), has at least one $\omega$-periodic solution in $\mathcal{R}$.

Proof. Let us define $H(\varphi, \mu): \mathcal{R} \times[0,1] \longrightarrow \mathcal{R}$ by

$$
H(\varphi, \mu)=\mu J \varphi
$$

Then, by using (26), we have

$$
\|H(\varphi, \mu)\|_{\mathcal{R}} \leq \mu\left(e^{\lambda \tau} \omega \widetilde{M}+e^{\lambda r} \sum_{t+\theta \leq t_{j}<t+\theta+\omega} \bar{M}\right) .
$$

Then, for each $\mu \in(0,1)$ the set $S=\{\varphi: \varphi=H(\varphi, \mu)\}$ is bounded. Since $J$ is completely continuous, then by using Schäffer's fixed point theorem, the Poincaré operator $J$ admits a fixed point.

Next, we give the conditions of the existence and uniqueness of a $\omega$-periodic solution of (1)-(3).

Theorem 3. Let $f:[0, \omega+\tau] \times \mathcal{R} \rightarrow \mathbb{R}^{n}$ be a map satisfying (I) and (II), where $\omega \geq \tau$, and $h_{j}, j=1, \ldots, l$ are bounded and satisfy the condition (III), and there exist constants $\bar{H}_{j}, j=1, \ldots, l$, such that

$$
\left\|h_{j}(\varphi(0))-h_{j}(\psi(0))\right\| \leq \bar{H}_{j}\|\varphi-\psi\|_{\mathcal{R}} .
$$

If, there exists a constant $C<1$, such that

$$
\frac{K \omega e^{\lambda r}}{1-e^{-\lambda \omega}}+\frac{e^{\lambda r}}{1-e^{-\lambda \omega}} \sum_{t-r+\omega \leq t_{j}<t+\omega} \bar{H}_{j} \leq C,
$$

then, the nonlinear impulsive problem (1)-(3), has a unique $\omega$-periodic solution in $\mathcal{R}$.

Proof. Let $\varphi, \psi \in \mathcal{R}$ be two solutions of (1)-(3), i.e., $J \varphi=\varphi$ and $J \psi=\psi$. Assume $\phi \neq \psi$. We have

$$
\begin{aligned}
\|\varphi(\theta)-\psi(\theta)\|= & \|J \varphi(\theta)-J \psi(\theta)\|, \\
\leq & e^{\lambda r} \int_{t+\theta}^{t+\theta+\omega}|G(t, s)|\|f(s, \varphi)-f(s, \psi)\|_{\mathcal{R}} d s+ \\
& e^{\lambda r} \sum_{t-r+\omega \leq t_{j}<t+\omega}\left|G\left(t, t_{j}\right)\right| \| h_{j}\left(\varphi(0)-h_{j}(\psi(0)) \|,\right. \\
\leq & \left(\frac{K \omega e^{\lambda r}}{1-e^{-\lambda \omega}}+\frac{e^{\lambda r}}{1-e^{-\lambda \omega}} \sum_{t-r+\omega \leq t_{j}<t+\omega} \bar{H}_{j}\right)\|\varphi-\psi\|_{\mathcal{R}}, \\
\leq & C\|\varphi-\psi\|_{\mathcal{R}} .
\end{aligned}
$$

Hence 


$$
\begin{aligned}
\|\varphi-\psi\|_{\mathcal{R}} & \leq C\|\varphi-\psi\|_{\mathcal{R}} \\
& <\|\varphi-\psi\|_{\mathcal{R}} .
\end{aligned}
$$

This contradiction implies, the uniqueness of the $\omega$-periodic solution of (1)-(3).

\section{Conclusions}

The method described in this work presents new challenges for more investigation on more realistic models; such as the extension of the ascorbic acid model [12] and HIV model [13,14]. Taking into account the delay effect on respective compartments [23-25]. This kind of work, will need more investigation on modeling validation effort, keeping a close eye on the real life data in order to have a more realistic model. The explicit solutions presented in the technical Lemma 4 and methods of proving the existence of periodic solutions are very useful for further future investigations.

Funding: This research was funded by Deanship of Scientific Research at King Saud University, Grant No. RG-1435-079.

Acknowledgments: The authors would like to express their deep appreciation to the Deanship of Scientific Research at King Saud University for supporting this Research group No. (RG-1435-079). The authors profusely thank the referees for their valuable comments.

Conflicts of Interest: The authors declare no conflict of interest.

\section{References}

1. Hale, J. Theory of Functional Differential Equations; Springer: New York, NY, USA, 1977.

2. Milman, V.D.; Myshkis, A.D. On the stability of motion in the presence of impulses. Sib. Math. J. 1960, 1, 233-237. (In Russian)

3. Mil'man, V.D.; Myshkis, A.D. Random impulses in linear dynamical systems. In Approximate Methods of Solution of Differential Equations; Publishing House of the Academy of Sciences: Kiev, Ukraim, 1963; pp. 64-81. (In Russian)

4. Bachar, M.; Arino, O. Stability of a general linear delay-differential equation with impulses. Dyn. Contin. Discret. Impuls. Syst. Ser. A Math. Anal. 2003, 10, 973-990.

5. Bainov, D.D.; Barbanti, L.; Hristova, S.G. Method of quasilinearization for the periodic boundary value problem for impulsive differential-difference equations. Commun. Appl. Anal. 2003, 7, 153-170.

6. Bainov, D.D.; Hristova, S.G.; Hu, S.C.; Lakshmikantham, V. Periodic boundary value problems for systems of first order impulsive differential equations. Differ. Integr. Equ. 1989, 2, 37-43.

7. Bainov, D.D.; Simeonov, P.S. Systems with Impulse Effect. Stability, Theory and Applications; Ellis Horwood Limited: Hemel Hempstead, UK; John Wiley \& Sons: Chichester, UK, 1989.

8. Bajo, I.; Liz, E. Periodic boundary value problem for first order differential equations with impulses at variable times. J. Math. Anal. Appl. 1996, 204, 65-73. [CrossRef]

9. Berezansky, L.; Braverman, E. Impulsive equations: Overview and open problems. Funct. Differ. Equ. 2008, 15, 39-56.

10. Pandit, S.G.; Deo, S.G. Differential Systems Involving Impulses; Springer: New York, NY, USA; Berlin, Germany, 1982.

11. Zhao, K. Global exponential stability of positive periodic solution of the n-species impulsive Gilpin-Ayala competition model with discrete and distributed time delays. J. Biol. Dyn. 2018, 12, 433-454. [CrossRef] [PubMed]

12. Bachar, M.; Raimann, J.G.; Kotanko, P. Impulsive mathematical modeling of ascorbic acid metabolism in healthy subjects. J. Theor. Biol. 2016, 392, 35-47. [CrossRef] [PubMed]

13. Miron, R.E.; Smith, R.J. Resistance to protease inhibitors in a model of HIV-1 infection with impulsive drug effects. Bull. Math. Biol. 2014, 76, 59-97. [CrossRef] [PubMed] 
14. Miron, R.E. Impulsive Differential Equations with Applications to Infectious Diseases. Ph.D. Thesis, Department of Mathematics and Statistics, Faculty of Science, University of Ottawa, Ottawa, ON, Canada, 2014.

15. Pan, T.; Jiang, D.; Hayat, T.; Alsaedi, A. Extinction and periodic solutions for an impulsive SIR model with incidence rate stochastically perturbed. Phys. A 2018, 505, 385-397. [CrossRef]

16. Li, J.; Nieto, J.J.; Shena, J. Impulsive periodic boundary value problems of first-order differential equations. J. Math. Anal. Appl. 2007, 325, 226-236. [CrossRef]

17. Nieto, J.J. Basic theory for nonresonance impulsive periodic problems of first order. J. Math. Anal. Appl. 1997, 205, 423-433. [CrossRef]

18. Bachar, M.; Magal, P. Existence of Periodic Solution for a Class of Delay Differential Equations with Impulses; Topics in Functional Differential and Difference Equations (Lisbon, 1999); Fields Inst. Commun. Amer. Math. Soc.: Providence, RI, USA, 2001; Volume 29, pp. 37-49.

19. Schäffer, H. Über die Methode der a priori-Schranken. Mathematische Annalen 1955, 129, 415-416. [CrossRef]

20. Deimling, K. Nonlinear Functional Analysis; Springer: New York, NY, USA, 1985.

21. Evans, L.C. Partial Differential Equations, Graduate Studies in Mathematics; American Mathematical Society: Providence, RI, USA, 2010.

22. Smart, D.R. Fixed Point Theorems; Cambridge Tracts in Mathematics, No. 66; Cambridge University Press: London, UK; New York, NY, USA, 1974.

23. Morse, S.S. Factors in the emergence of infectious diseases. Emerg. Infect. Dis. 1995, 1, 7-15. [CrossRef] [PubMed]

24. Wang, G.; Yin, T.; Wang, Y. In vitro and in vivo assessment of high-dose vitamin C against murine tumors. Exp. Ther. Med. 2016, 12, 3058-3062. [CrossRef] [PubMed]

25. Yung, S.; Mayersohn, M.; Robinson, J.B. Ascorbic acid absorption in humans: A comparison among several dosage forms. J. Pharm. Sci. 1982, 71, 282-285. [CrossRef] [PubMed]

(C) 2019 by the author. Licensee MDPI, Basel, Switzerland. This article is an open access article distributed under the terms and conditions of the Creative Commons Attribution (CC BY) license (http:// creativecommons.org/licenses/by/4.0/). 